\title{
El dinero en la historia del pensamiento económico: la teoría monetaria post-keynesiana y su confrontación con la ortodoxia
}

\author{
Esteban CRUZ HIDALGO \\ Universidad de Extremadura \\ ecruzhid@unex.es \\ Francisco Manuel PAREJO MORUNO \\ Universidad de Extremadura \\ fmparejo@unex.es
}

Received: 30/11/2015

Accepted: 13/04/2016

\begin{abstract}
Resumen
Las disputas en torno a determinados aspectos del dinero, como su neutralidad y el carácter endógeno o exógeno de la oferta monetaria, han sido permanentes entre las distintas escuelas de pensamiento y autores, estando su origen, probablemente, en la época de desarrollo del pensamiento escolástico. En este artículo pretendemos, en primer lugar, realizar un recorrido cronológico e histórico sobre el tratamiento científico económico del dinero, para, en segundo lugar, poner sobre la mesa la macroeconomía ortodoxa a la que han dado lugar las interpretaciones al respecto, así como los enfoques alternativos frente a este pensamiento dominante. Finalmente, intentamos poner en valor los desarrollos monetarios post-keynesianos, integrados en lo que denominan "Economía Monetaria de Producción", confrontándolos con la llamada Nueva Síntesis Neoclásica.
\end{abstract}

Palabras clave: dinero, pensamiento económico, endogeneidad del dinero, neutralidad monetaria

\begin{abstract}
Disputes over certain aspects of money, as its neutrality and the endogenous or exogenous nature of the money supply, have been ongoing between the various schools of thought and authors, its origin probably being at the time of development of scholastic thought. In this paper we intend, firstly, to make a chronological and historical overview about money, with the objective of highlighting the monetary developments of the orthodox macroeconomics, as well as the alternative approaches against this dominant thought. Finally, we try to value the post-Keynesian monetary developments, which are integrated into what is called "Monetary Economy of Production", confronting them with the so-called New Neoclassical Synthesis.
\end{abstract}

Keywords: money, economic thought, endogenous money, neutrality of money

JEL Classification: B19, B25, B50

\section{Introducción}

Las controversias sobre el papel del dinero en la economía han sido permanentes entre las distintas escuelas de pensamiento y autores, estando su origen, probablemente, en la época de desarrollo del pensamiento escolástico. Algunos de los pensadores que podemos situar en esta corriente medieval ya bosquejaban una primitiva "teoría cuantitativa del dinero", al asociar vagamente las variaciones en los precios de las mercancías con la entrada de oro y plata en Europa procedente de las colonias españolas de América.

Este planteamiento exógeno del dinero derivaría de la supuesta e histórica vigencia en el mundo de un sistema dinero-mercancía, en el que la base monetaria de los países podía verse alterada por el intercambio comercial (esto es, por la existencia de flujos internacionales de metales preciosos entre las naciones) o por el descubrimiento de nuevos yacimientos de oro, siendo, por tanto, las variaciones de la cantidad de dinero en circulación no susceptibles de manipulación política ni controlables directamente por los agentes, esto es, exógenas al sistema. 
De igual modo, en un sistema capitalista como el actual, donde prevalece el dinero fiat o fiduciario, cuyo valor se sustenta en una relación de confianza en la institución emisora (al no tener dicho dinero como contrapartida un determinado montante de metales preciosos), y donde opera el multiplicador monetario a través del control de reservas por parte del Banco Central, la exogeneidad vendría determinada en cuanto a que los bancos actúan como simples agentes pasivos, intermediando entre los depósitos de los ahorradores y los préstamos a sus clientes, y también en cuanto a que el Banco Central es capaz de controlar tanto a corto como a largo plazo la base monetaria.

A pesar de ser cuestionable esta postura, o eso pensamos, constituye la visión predominante sobre el dinero en los manuales de macroeconomía, los cuales mantienen con profusión el legado histórico y artificial del dinero-mercancía y del patrón oro, lo que entendemos es un fatal malentendido para la comprensión de la importancia del dinero para la producción, y por tanto, para el empleo. Y en definitiva para percibir su capacidad de alterar las variables reales de la economía, y no solo de modificar las variables nominales, o sea los precios, como sostienen las posturas más ortodoxas.

La cuestión adquiere mayor complejidad cuando analizamos una moderna economía de crédito, donde los bancos comerciales pueden ser contemplados como agentes creadores de dinero a través de sus operaciones de préstamo, no siendo, por tanto, meros intermediarios entre el ahorro individual y la inversión. En un sistema de estas características, la oferta monetaria es necesariamente endógena, estando dirigida por la demanda de créditos y no por las restricciones que dependen de la oferta y de las dotaciones de dinero existentes fruto del ahorro previo.

Esta concepción está presente de manera indiscutible en el núcleo de todas las corrientes post-keynesianas e implica que el dinero nunca puede ser neutral (Lavoie, 1984). Su admisión llevaría a considerar que, en una Economía Monetaria de Producción, con un sistema bancario creador de dinero fiduciario-crédito, las empresas toman prestado para financiar el proceso productivo, reembolsando el préstamo cuando el output es vendido mediante la entrega al prestamista de los depósitos acumulados, saldando así la deuda previamente contraída. En definitiva, en esta óptica el dinero tendría un origen claramente endógeno y un carácter nítidamente no neutral, habida cuenta de su capacidad de dinamizar la producción y el empleo.

La principal contribución de este trabajo es la confrontación de la visión ortodoxa del dinero, exógena y neutral, con los nuevos desarrollos monetarios del análisis post-keynesiano, los cuales, a pesar de ser ya notables, siguen sin adquirir gran notoriedad en los manuales de teoría económica. Asimismo, el trabajo puede servir, en nuestro criterio, para rescatar la controversia original que existía en la obra de los economistas clásicos en torno al dinero, la cual se ha ido perdiendo a lo largo del tiempo; algo que, a tenor de la evolución del sistema bancario y de su creciente protagonismo en la economía, no deja de ser llamativo. El hecho de centrar el análisis en la crítica de la exogeneidad del dinero y su neutralidad contribuye, en nuestra opinión, a reavivar este debate controvertido en un momento en que la política monetaria ha pasado a un primer plano, y con ella la vigencia de los desarrollos teóricos relativos al dinero.

Para abordar el propósito anterior, en el apartado 2 realizamos un recorrido cronológico e histórico sobre la cuestión de la exogeneidad o endogeneidad de la oferta monetaria, donde cada planteamiento contiene la visión de un tipo de sistema: por un lado, una economía de intercambios reales o trueque donde impera el dinero-mercancía o, en un estadio más avanzado, el dinero fiduciario; y por el otro, una economía monetaria de producción con dinero fiduciario-crédito, que presupone una función primordial de los bancos en la financiación del proceso de producción.

Del mismo modo, en el apartado 3 exponemos cómo la macroeconomía ortodoxa, adscrita a la hipótesis de exogeneidad de la oferta monetaria y a la dependencia del dinero de factores reales, persiste en la denominada dicotomía clásica, concediendo una influencia limitada al dinero en el corto plazo.

Opuesto a esto, en el apartado 4 intentamos poner en valor los desarrollos monetarios postkeynesianos, que contemplan la oferta de dinero como endógena y el dinero no neutral, tanto a corto como a largo plazo, visión que trataremos de confrontar con el enfoque de la llamada Nueva Síntesis Neoclásica.

Finalmente, en el apartado 5 realizamos algunas reflexiones a modo de conclusión. 


\section{El legado del patrón dinero-mercancía: un repaso bibliográfico}

Un asunto recurrente en el análisis del papel del dinero que realiza la teoría económica moderna es la disyuntiva entre aquellos que piensan que los cambios exógenos en la oferta de dinero juegan un papel causal sobre la variación del nivel de precios, sin afectar a las variables reales, frente a aquellos otros que defienden que las variaciones en la oferta monetaria son endógenas, respondiendo a los cambios en la actividad económica, permitiendo de este modo financiar el comienzo de nuevos procesos de producción. ${ }^{1}$.

Es conocida la concepción neutral del dinero de la mayor parte de los autores clásicos, expresada con especial vehemencia por Juan Bautista Say en su magistral Tratado (1803), y de forma más concisa en el fragmento titulado De los mercados que, de este autor, se reproduce en Segura y Rodríguez Braun (1998). Esta concepción del dinero, como mero agilizador de las transacciones (como "vehículo para transportar el valor de las cosas", decía Say), no se aleja en demasía del denominado "análisis real" de Schumpeter, el cual toma el dinero como un "velo" adoptado simplemente para facilitar las transacciones de los costes asociados a una economía de trueque, sin reconocérsele la más mínima capacidad de influencia sobre la producción y el empleo (Schumpeter, 2012). Esta sería, por tanto, y con matices, la visión dominante entre finales del siglo XVIII y comienzos del siglo XX, que difiere sustancialmente de la existente durante el período mercantilista previo.

En este sentido, los análisis realizados por autores mercantilistas en los siglos XVII y XVIII conciben el proceso económico como resultante de la sucesión de flujos de gasto, donde, por ejemplo, hasta el gasto improductivo de los terratenientes (en vicios y lujos) contribuye al funcionamiento del sistema económico. ${ }^{2} \mathrm{Si}$ exceptuamos a los autores escolásticos antes aludidos, hay que remontarse a David Hume (1752) para encontrar una enunciación que introduzca la noción de una relación causal entre la oferta de dinero y los precios, en un contexto de dineromercancía.

En la exposición de su "mecanismo de flujo especie-precio", Hume argumenta la relación directa entre la oferta monetaria y los precios, admitiendo la existencia de efectos reales (temporales o transitorios, esto es, a corto plazo) cuando hablamos de economías abiertas, cuestión ésta que ha sido pasada por alto por la mayoría de los economistas clásicos. Sí ha sentado cátedra su alusión a que, en el largo plazo, estos efectos sobre la producción (inducidos por el comercio exterior, en su mayor parte) se verían compensados, garantizándose el equilibrio de la balanza comercial de las distintas naciones a través de la regulación de los niveles de precios y, dicho sea de paso, desterrando para siempre el principio mercantilista de la posibilidad de mantener de forma permanente una balanza comercial positiva (Blaug, 1986).

Aún más lejos que Hume, en cuanto a los efectos que un aumento de la cantidad de dinero proyectaba sobre la producción, había llegado Cantillon (1755). En su Essai describió una secuencia de gastos por los cuales un aumento en la proporción de dinero en circulación, originario de las minas de oro y plata que se encuentran en un Estado, aumentaría la demanda de quienes se beneficiaran directamente de la riqueza de las minas, que con sus gastos darían trabajo a quiénes antes carecían de él. Las reacciones que el aumento de los precios relativos provocaba en unos y otros para mantener su nivel de consumo, principalmente de los terratenientes, llevarían a una reducción del número de habitantes en el Estado, permitiendo un mayor consumo a quienes se quedaran. Tal observación parece sugerir una primitiva noción de no neutralidad tanto a corto como a largo plazo.

El circuito de gastos mencionado y sus efectos, o como se ha denominado posteriormente, el "efecto Cantillon", que supone un aumento progresivo de los precios conforme el gasto va ex-

\footnotetext{
${ }^{1}$ Una síntesis bibliográfica de los trabajos que han abordado este asunto puede obtenerse de Blaug (1985), Roncaglia (2006), Hein (2008), Amon (2011) y Schumpeter (2012).

${ }^{2}$ Este y otros principios mercantilistas fueron repudiados por Smith en su obra culmen (1776), la cual es un claro alegato contra la doctrina mercantilista en general y contra el gasto en particular, constituyendo una reafirmación de la ética protestante que ensalza la conducta frugal e industriosa.
} 
tendiéndose por la economía, va intrínsecamente unido a la concepción ochocentista de la teoría del valor-tierra (Cantillon 1755). Al igual que hicieran posteriormente los fisiócratas y sus contemporáneos, Cantillon suponía que la principal fuente de riqueza es la tierra y el principio de todo gasto depende de su fertilidad (Quesnay, 1759; Turgot, 1766). Solo si aceptamos que la tierra produce una cantidad fija de productos, los efectos sobre los precios de un aumento del dinero en circulación se sostienen lógicamente. No obstante, debe valorarse que los rendimientos de la agricultura se entienden decrecientes, en consonancia con la vigencia en la época de una economía de escasez con apenas progreso tecnológico. Esta situación, obviamente, no se produce en la actualidad, donde las restricciones reales se presentan en el factor trabajo y no en el factor tierra. Aunque esta no ha sido la línea seguida por economistas ulteriores.

En otro orden y siguiendo la herencia humeana, deudora, como el propio Hume reconocía, de las aportaciones monetarias liberales de John Locke, ${ }^{3}$ los economistas clásicos asumieron como propia la ortodoxia de la "teoría cuantitativa" y comenzaron a desarrollar los fundamentos del análisis real, tal y como aparece en la obra de Smith. A saber, como consecuencia del libre albedrío de los individuos; y estando movidos éstos por su egoísmo, una suerte de mano invisible establecería los precios relativos definidos como tasas de sustitución entre bienes físicamente determinados, equilibrando simultáneamente todos los mercados de forma natural.

En esta concepción clásica, el dinero solo entraría en la economía como un simple medio de cambio para facilitar las transacciones, permitiendo superar las barreras inherentes al trueque, donde los deseos del comprador y vendedor deben coincidir exactamente para poder hacer efectivo el intercambio. ${ }^{4}$ En cuanto a la oferta de dinero, ésta vendría dada por el acervo de metales preciosos existente, por lo cual el uso improductivo del ahorro en la producción de bienes y servicios que satisfagan los vicios de los terratenientes es contemplado como un impedimento tan grave para el desarrollo económico como la insuficiencia del propio ahorro.

Obviamente, bajo este prisma, los bancos son esencialmente prestamistas, o si se prefiere, intermediarios que hacen circular el dinero de las personas. De esta forma, no se admite que la emisión de billetes por encima de las reservas líquidas constituya una creación efectiva de oferta monetaria, sino un mecanismo para aumentar su velocidad de circulación con la finalidad de hacer más eficaz su uso.

Ante la visión generalmente aceptada que se ha presentado en los párrafos anteriores, no hubo, sin embargo, unanimidad plena. Ya a comienzos del siglo XVIII, el economista escocés John Law (a quien se atribuye la invención del papel moneda) argumentó que los bancos, al prestar varias veces las mismas sumas de dinero, ejercían una función implícita de creación de dinero, advirtiendo que la velocidad de circulación no aumentaba en absoluto, al depender de factores institucionales. Por este motivo se consideraba, de hecho, una variable estable en las formulaciones de la "teoría cuantitativa clásica" (Law, 1705).

Casi un siglo después de las "consideraciones" de Law tuvo lugar, en el marco temporal de las guerras napoleónicas, el debate entre bullonistas y anti-bullonistas. ${ }^{5}$ La suspensión de la

\footnotetext{
${ }^{3}$ En el ámbito monetario, las aportaciones de Locke se sintetizan en su escrito Some considerations of the consequences of the lowering of interest, and raising the value of money (1691), en el cual se ponen de manifiesto las distorsiones monetarias ocasionadas por la modificación artificial del tipo de interés por parte de los estados y su posición contraria a tal modificación, por tanto.

${ }^{4}$ Smith mantiene un enfoque metalista a lo largo de La riqueza de las naciones (1776), con un apoyo a la emisión de billetes fijados a la convertibilidad en oro y plata, y que fue el camino seguido por sus continuadores. Pese a ello bosqueja un planteamiento alternativo en la parte que analiza los sistemas monetarios introducidos en las colonias inglesas de América del Norte. Este "chartalismo embrionario" en Smith aparece en su observación del papel moneda como creación del Estado, cuya aceptación para el pago de impuestos le da valor. Además, llega a especular con la posibilidad de un sistema bancario donde el dinero creado sea una señal de confianza y no esté basado en la cantidad de metales preciosos. Para una revisión más detallada de ello ver Giacomin (2007).

${ }^{5}$ Este debate enfrentaba a los primeros, defensores a ultranza de un sistema monetario estrictamente metálico como mejor forma de control de los precios, frente a las posturas anti-bullonistas, más proclives al establecimiento de un sistema monetario fundamentado en el papel moneda, con autonomía monetaria por parte de los bancos emisores. Véase al respecto Blaug (1985), Roncaglia (2006) y Schumpeter (2012).
} 
convertibilidad en oro de los billetes del Banco de Inglaterra en 1797 y la inflación de la época llevaron a los primeros a defender la no emisión de papel moneda por parte de éste, al vincular la subida de precios con el aumento de dinero en circulación, y no con circunstancias como la escasez relativa de bienes en esta coyuntura bélica, que era el argumento ofrecido por los antibullonistas.

En nuestro criterio, una de las contribuciones más relevantes a este debate la ofreció Thornton (1802), al sugerir la adopción por parte del Banco de Inglaterra de la denominada "Regla de Palmer", que admitía la expansión del crédito como el causante de la salida de oro del banco central. Como un aumento de la oferta de dinero estimula la demanda de préstamos, aumentando progresivamente el precio de los bienes de inversión, la tasa de interés aumentará, automáticamente, para contrarrestar el menor rendimiento esperado de la inversión. El aumento del tipo de interés disminuiría la cantidad de préstamos, lo que se traduciría en un menor volumen de transacciones y de la ocupación, con el consiguiente descenso de los precios.

Esta idea la planteó por primera vez Cantillon (1755) cuando advirtió que la velocidad de circulación del dinero era equivalente al aumento de su cantidad, infiriendo de ello que las medidas destinadas a disminuir su velocidad de circulación compensarían los efectos en los aumentos de los precios por el aumento de la oferta monetaria.

El debate sobre los problemas monetarios se reavivó años más tarde culminando con la adopción de la "Ley de Peel" en 1844, ${ }^{6}$ y con la confrontación entre la Currency School y la Banking School sobre el funcionamiento de los bancos. ${ }^{7}$ Los partícipes de la Banking School intervinieron negando la posibilidad de una excesiva emisión de papel moneda, porque las necesidades del comercio controlarían el volumen de los billetes emitidos, por lo cual no era necesario la adopción de ninguna regla mientras se mantuviese la convertibilidad. Por el contrario, los defensores de una moneda puramente metálica, integrados en la Currency School, pedían una regla que operase como un mecanismo por el cual una moneda mixta de oro y papel variase de la misma forma que un sistema de dinero-mercancía puro.

El debate se saldó con la mencionada "Ley de Peel", que separó las operaciones bancarias de la emisión de la moneda, lo que impuso un requisito de reservas del ciento por ciento para los billetes del banco. Los partidarios de la Banking School sostuvieron que el uso de depósitos bancarios, letras de cambio y otras formas de crédito como sustitutos de los billetes del banco, frustrarían la intención de controlar la oferta monetaria por parte del banco central.

\section{La construcción de la Nueva Síntesis Neoclásica}

Los fundamentos de la teoría monetaria neoclásica, que siguiendo la tradición clásica se posicionan dentro del análisis real, contienen una mezcla de elementos neo-walrasianos y neowicksellianos. ${ }^{8}$ La trascendental influencia de ambos autores se materializa en aspectos centrales del paradigma neoclásico como el equilibrio general y la existencia de una tasa de interés natural.

A diferencia de los otros autores corresponsables de lo que hoy conocemos como revolución marginalista, ${ }^{9}$ Walras (1877) elaboró un esquema de ecuaciones basado en un modelo de trueque perfecto, en el cual no había asignado rol alguno al dinero, puesto que no era necesario para conseguir el equilibrio general de los precios relativos de todos los bienes. Los precios eran

\footnotetext{
${ }^{6}$ La conocida "Ley de Peel" fue una legislación bancaria impulsada por el primer ministro británico Robert Peel cuyo rasgo característico fue el establecimiento de un coeficiente de caja del 100\% en oro para los bancos privados, respecto a la emisión de papel moneda que realizaban.

${ }^{7}$ Trabajos como Blaug (1985), Roncaglia (2006) y Schumpeter (2012) analizan esta confrontación decimonónica de forma sintética y clara.

${ }^{8}$ Una profunda exposición de los fundamentos neo-walrasianos y neo-wicksellianos se puede ver en Rogers (1989).

${ }^{9}$ Hablamos del austriaco Carl Menger y del inglés William Stanley Jevons, descubridores, junto a Walras, del concepto de utilidad marginal.
} 
simultáneamente "negociados" a través del imaginario proceso del "tâtonnement" ", llevado a cabo por un subastador imaginario que aparece como recolector centralizado de la información dispersa. Tal artificio permite juntar el principio y el final de un periodo de producción en un mismo momento de tiempo, posibilitando conocer los beneficios esperados antes de que haya comenzado la producción. Así es como este esquema cumple escrupulosamente con la denominada "Ley de Say", conocida también como "Ley de los mercados", que podemos asimilar, en última instancia, a la "Ley de Walras".

Con el asentamiento del dinero fiduciario con la emisión de billetes ligados a la cantidad de oro que mantenía en reservas el Banco Central, Wicksell (1898) se propuso extender la aplicación del equilibrio a aspectos monetarios, valiéndose de la Teoría Cuantitativa del Dinero, que sería luego sintetizada por Fisher (1911) en la famosa "ecuación de cambio". ${ }^{11}$

La contribución principal de Wicksell fue el distinguir entre dos tipos de interés: un tipo de interés real o natural, que se correspondía con la productividad marginal del capital y la tasa de preferencia intertemporal de los hogares y equilibraba la oferta y demanda de fondos prestables $;{ }^{12}$ y un tipo de interés de mercado o bancario, fijado por las autoridades monetarias. La divergencia entre ambos tipos de interés le valió para dar una explicación de los ciclos económicos y de las oscilaciones sobre el nivel general de precios, el cual únicamente podría mantenerse estable si la oferta monetaria aumentaba al mismo ritmo que la productividad. Esta introducción del ahorro y la inversión en la estructura del equilibrio general no afecta al cumplimiento de la Ley de Say, puesto que supone que el ahorro es una manera indirecta de gastar, manteniéndose, por tanto, una de las implicaciones básicas de la teoría clásica; esto es, que el ahorro y la inversión agregados se equilibran mediante el tipo de interés.

Para Fisher, la ecuación de la teoría cuantitativa solo era válida en equilibrio, no para los periodos de transición donde había influencias indirectas en el poder de compra, como detalla el proceso acumulativo descrito por Wicksell, por lo cual el dinero para ambos autores es considerado como no neutral. La posibilidad de que un leve movimiento más allá del punto de equilibrio pudiese desencadenar fuerzas que llevaran a la economía a otra situación diferente de su equilibrio original, tal como se expone en su hipótesis de la deflación de la deuda (1933), no cristalizó entre los economistas posteriores, o al menos no tanto a como lo hizo antes de la Gran Depresión ${ }^{13}$.

La distinción de Wicksell entre tipo de interés natural, derivado de la productividad marginal del capital, y tipo de interés de mercado, de cuyas discrepancias surge el nexo entre los sectores real y monetario, se encuentra en el modelo IS-LM (Rogers, 1989, p. 279) ${ }^{14}$ que ha acaparado el terreno de la macroeconomía desde Keynes (1936) hasta nuestros días. ${ }^{15}$

Este modelo, que debemos atribuir a la lectura particular que de la "Teoría General" hizo Hicks (1937), difiere de la estructura clásica en la incorporación de la teoría de la preferencia de la liquidez; del ahorro como una función del ingreso en lugar de la tasa de interés; y de la presencia de rigidez salarial. Estos tres elementos llevan a la proposición de una política monetaria expansiva que de manera transitoria influya en la marcha de la economía hacia el equilibrio, buscando explotar la existencia de un trade-off entre inflación y desempleo, tal como sugiere la regularidad empírica mostrada por la Curva de Philips.

\footnotetext{
${ }^{10}$ Rogers (1989) describe de forma simple el funcionamiento de este mecanismo walrasiano de "prueba y error" hacia el equilibrio.

${ }^{11}$ En ella $(M V=P T)$, Irvin Fisher se limita a relacionar el nivel de precios $(\mathrm{P})$ directamente con la cantidad de dinero en circulación (M), por un lado, y con su "eficiencia" o velocidad (V), por el otro, e inversamente con el volumen (físico) de transacciones $(\mathrm{T})$ que se producen en una economía determinada. ${ }^{12} \mathrm{La}$ oferta de fondos prestables proviene de los hogares que quieren ahorrar parte de su ingreso y prestarlo. La demanda de fondos prestables proviene de los hogares y las empresas que quieren pedir prestado para invertir.

${ }^{13}$ Keen (2015) aborda de forma analítica las aportaciones realizadas por Fisher poniendo de manifiesto la notable diferencia en los planteamientos del autor antes y después del crack de 1929.

${ }^{14}$ Una explicación precisa de esta cuestión se encuentra en el capítulo 4 de este libro.

${ }^{15}$ Anisi (2005) presenta la evolución de la macroeconomía en el siglo XX a través de un modelo IS-LM correspondiente a cada una de las diferentes corrientes.
} 
Después, los monetaristas emergieron y cargaron contra la Curva de Phillips, tomándola como vertical en el largo plazo, al desaparecer la ilusión monetaria, argumentando que los efectos a corto plazo de aumentar la masa monetaria serían efectivos únicamente si la inflación no fuese anticipada por los agentes económicos. ${ }^{16}$

La hipótesis de expectativas racionales aparece así como un elemento fundamental para mantener el supuesto de neutralidad del dinero, desarrollada ampliamente por la Escuela del Ciclo Real (Nuevos Clásicos) desde los años 80, la cual plantea que los agentes económicos consiguen anticipar correctamente la política monetaria de manera sistemática, ${ }^{17}$ sustituyendo la curva de Philips por la NAIRU. ${ }^{18}$ A diferencia del modelo IS-LM, este enfoque surge formalmente de la optimización por parte de las empresas y consumidores, reimplantando los fundamentos microeconómicos pre-keynesianos como base de su análisis. ${ }^{19}$

Confrontando la hipótesis de la existencia de ajustes instantáneos, pero continuando con una misma macroeconomía de agente representativo con base microeconómica, aparece una nueva corriente de pensamiento keynesiana. Los Nuevos Keynesianos rechazan la hipótesis de ajustes instantáneos adoptada por los Nuevos Clásicos, abandonando el análisis de las interdependencias entre mercados. Su rechazo a la hipótesis de ajustes instantáneos se formaliza en la existencia de un estado de equilibrio general imperfectamente competitivo, derivado de ciertas imperfecciones en los mercados y otras rigideces reales tomadas de la nueva microeconomía, como la información asimétrica. ${ }^{20}$

Estas diferencias centran la disputa entre estas dos corrientes predominantes, coincidentes, eso sí, en la existencia de tales rigideces. No obstante, para los Nuevos Clásicos éstas son exógenas a los mercados y vendrían derivadas de las decisiones institucionales; mientras que para los Nuevos Keynesianos, las rigideces responderían al comportamiento de los agentes ante imperfecciones inevitables.

Con estos fundamentos aparecería más tarde lo que se conoce como Nueva Síntesis Neoclásica, combinando los puntos de disputa de los enfoques de los Nuevos Clásicos y de los Nuevos Keynesianos. ${ }^{21}$ Los modelos de Equilibrio General Dinámico Estocástico, o DSGE, ${ }^{22}$ se construyen con salarios o precios rígidos, basándose las decisiones de los consumidores y la fijación de precios por parte de las empresas en la hipótesis de expectativas racionales. En estos modelos el dinero no juega ningún papel más que el de proporcionar una unidad de cuenta. ${ }^{23}$

Tal forma de proceder ofrece ciertas recomendaciones de política económica con el objetivo de influir en la demanda agregada a corto plazo, ajustándola a la tendencia de la actividad real o brecha de producción, que queda en este marco determinada por factores del lado de la oferta. La existencia de imperfecciones y rigideces hace que las fluctuaciones de la demanda agregada no se compensen automáticamente por los movimientos en la tasa de interés, llevando a los Bancos Centrales a tratar de corregir tal brecha mediante la política monetaria, cuyo principio

\footnotetext{
${ }^{16}$ La mejor exposición del enfoque monetarista puede verse en Friedman(1987).

${ }^{17}$ Para profundizar sobre la cuestión de la neutralidad del dinero entre las diferentes escuelas neoclásicas ver Jijón (2000) y Giraldo (2006).

${ }^{18}$ Acrónimo derivado de la expresión inglesa Non-Accelerating Inflation Rate of Unemployment. Como es sabido, la NAIRU constituye un desarrollo que surge de la modificación de la Curva de Phillips.

${ }^{19}$ Para un tratamiento más conciso de los fundamentos microeconómicos de la Nueva Macroeconomía Clásica ver Deleplace (2008). Ver también Rogers (2013) para una crítica al concepto de "ilusión monetaria".

${ }^{20}$ Sobre las hipótesis auxiliares añadidas por los Nuevos Keynesianos al marco de los Nuevos Clásicos, ver Ospina y García (2009).

${ }^{21}$ En los trabajos de Blanchard (2009) y Woodford (2009) se exponen de forma detallada los puntos clave de la Nueva Síntesis Neoclásica.

${ }^{22}$ Dynamic Stochastic General Equilibrium.

${ }^{23}$ Ver Gali y Gertler (2007) para las características principales de los modelos de la Nueva Síntesis Neoclásica.
} 
operacional es la regla de Taylor. ${ }^{24}$ Con ello, las autoridades monetarias alteran la oferta monetaria operando indirectamente a través de la fijación del tipo de interés real a corto plazo, que se mantiene en el nivel elegido mediante operaciones de mercado abierto, esto es con la compraventa de títulos públicos para expandir o contraer el montante de reservas del sistema bancario (Taylor, 2008). ${ }^{25}$

La manipulación del tipo de interés al que el Banco Central ofrece reservas al sistema bancario se corresponde más con una explicación endógena de la oferta monetaria, al contrario que la explicación exógena que surge de la literatura económica neoclásica. ${ }^{26}$ Pese a esta forma de obrar, que plantea la actividad del Banco Central como una función de respuesta a la petición de reservas de los bancos privados, la literatura neoclásica sigue explicando la determinación de la tasa de interés por la oferta y demanda de fondos prestables, y por tanto, manteniendo una explicación exógena de la oferta monetaria como una variable controlada por el Banco Central a través del control de las reservas obligatorias.

A día de hoy se sigue explicando el proceso por el cual los bancos conceden créditos como un proceso acumulativo que permite multiplicar la oferta de dinero a partir de la existencia precedente de ahorro por parte de los depositantes. Los bancos aceptarían recursos de los ahorradores creándose los depósitos, a partir de los cuales operaría el multiplicador monetario, siendo los créditos limitados por la cantidad de reservas necesarias impuestas por el Banco Central. ${ }^{27}$ Esto es lo que se conoce como sistema de reserva fraccionaria, tal y como se enseña en los libros de texto. ${ }^{28}$

Así, estos teóricos creen que un mayor ahorro, como consecuencia de un menor consumo y déficits públicos más bajos, daría lugar a un suministro de fondos más elevado, tasas de interés más bajas, más inversión y con ello un mayor crecimiento. En ausencia de cualquier fricción institucional, el dinero volvería a ser un "velo" sobre las variables reales, siendo únicamente las imperfecciones y asimetrías las que permiten al Banco Central influir sobre las variables reales a través del tipo de interés real a corto plazo.

Del mismo modo, el paradigma conocido como Escuela Austriaca de Economía defiende su versión particular sobre el dinero como pieza que permite el intercambio indirecto en el tiempo, aludiendo a las preferencias temporales entre consumo de bienes presentes y futuros de los individuos ${ }^{29} \mathrm{Si}$ se expandiese la oferta de fondos prestables sin respaldo previo de ahorro, la distorsión del tipo de interés natural wickselliano provocaría perniciosos efectos sobre la inversión, causando descoordinación en las decisiones intertemporales de productores y consumidores. Esta es la esencia de lo que se conoce como "teoría del ahorro forzoso" o "efecto expulsión", que queda ilustrada en el no consumo real derivado de las variaciones de los precios a consecuencia de una expansión de la demanda por la expansión del crédito bancario. Nuevamente tenemos aquí la defensa del análisis real caracterizado por una economía de trueque, donde los cambios en la oferta de dinero son una distorsión exógena.

Sin embargo, en una teoría monetaria de producción caracterizada por la endogeneidad de la oferta monetaria, como exponemos en el siguiente punto, un aumento de los fondos ahorrados y

\footnotetext{
${ }^{24}$ Esta regla sugiere que el Banco Central puede controlar la inflación por la vía de incrementar los tipos de interés reales en torno al doble que el aumento de la inflación.

${ }^{25}$ Sobre este planteamiento, autores como Friedman y Kuttner (2010) apuntan que los cambios institucionales ocurridos en las últimas décadas han hecho que los Bancos Centrales hoy día puedan hacer variar el tipo de interés del mercado interbancario sin ni siquiera tener que modificar el volumen de reservas del sistema bancario, a través de lo que se conoce como el "efecto anuncio". Desde esta óptica, los cambios en el tipo de interés que controla el Banco Central no llevarían aparejados cambios en la oferta monetaria, ni tampoco cambios significativos en la base monetaria.

${ }^{26}$ Una crítica minuciosa sobre la Nueva Síntesis Neoclásica puede verse en Arestis y Sawyer (2008) y Fontana y Pasarella (2014).

${ }^{27}$ Para una crítica detallada de la teoría de los fondos prestables, ver Lindner (2015).

${ }^{28}$ Ver Häring (2013) para una crítica sobre cómo los libros de texto exponen el papel de los Bancos Centrales y los bancos privados en el sistema monetario.

${ }^{29}$ La literatura de esta escuela se enfoca principalmente a la Teoría del Ciclo Austriaco, cuyo desarrollo sigue el trabajo de Hayek (1931). Una exposición de esta teoría en Alonso, Bagus y Rallo (2011).
} 
sacados de la circulación por el motivo que sea, se traduce en un exceso de oferta de bienes y servicios, con efectos reales sujetos a un entorno de incertidumbre radical que inducen un descenso de la producción y el empleo y, finalmente, de los precios.

\section{La alternativa de una teoría monetaria de producción}

Mientras que en la economía ortodoxa el dinero tiene un papel irrelevante, integrándose en el intercambio en forma de stock como si fuese lanzado desde helicópteros, tal como evocase Friedman (1969), los economistas post-keynesianos toman la oferta monetaria como endógena e impulsada por la demanda, situando el dinero en el núcleo de su análisis como parte integral para el comienzo del proceso de producción, y prestando especial atención a las contrapartes de los préstamos otorgados por el sistema bancario. ${ }^{30}$.

El principio de demanda efectiva, esencial en la estructura de una Economía Monetaria de Producción, implica que las empresas demandarán crédito en función de las expectativas de realización de un beneficio monetario, por lo cual la oferta de dinero no puede ser establecida arbitrariamente por el Banco Central. De esta forma, el dinero nunca puede ser concebido como neutral. ${ }^{31}$

La idea básica, como señalase Kaldor (1970) en su famosa crítica al monetarismo, niega la correlación entre el crecimiento de la oferta monetaria y el nivel general de precios, como es establecido en la Teoría Cuantitativa. Contradiciendo la causalidad señalada por la ecuación de Fisher se postula una lectura inversa de la misma, siendo las fluctuaciones de la demanda, determinadas en última instancia por el crecimiento de la renta global, las que dan origen a los cambios en la oferta monetaria.

La mayor parte de las modernas controversias entre los economistas ortodoxos y los economistas post-keynesianos traen a colación los debates de la Currency School y la Banking School del siglo XIX, quienes, como vimos en el apartado 2, centraron su atención en el crédito como respuesta de los agentes privados para facilitar la producción y el comercio, en función de la demanda. Del lado de la Banking School se posicionó Marx (1885), quien en sus esquemas de reproducción del libro II de El capital proyecta un circuito de producción que comienza con el dinero y se basa en la expectativa de conseguir más dinero.

Para Marx, como para los autores post-keynesianos, el dinero es un fenómeno social que establece la forma específica de la lógica capitalista, basada en la acumulación del mismo. Sin embargo, la teoría marxista ha tenido hasta hace unos años poco que decir sobre cómo se crea el dinero y sobre el funcionamiento de los bancos, navegando en las aguas del análisis real (Sardoni, 2003), aunque no de forma tranquila, tal como sí ocurre en la economía neoclásica.

El rechazo de Marx a la Ley de Say (Marx, 1867) no planteaba más que la separación en el tiempo de acciones que incluyen bienes reales como consecuencia del intercambio indirecto; mientras que su noción de competencia entre capitalistas esboza un sistema capitalista de una naturaleza inherentemente inestable y en desequilibrio. La obstinación en la Ley de la Tasa de Ganancia Decreciente (LTGD) del análisis marxista ha llevado a este paradigma igualmente a defender la neutralidad del dinero a largo plazo, obviando los obstáculos a la realización de los beneficios. ${ }^{32}$

\footnotetext{
${ }^{30}$ Ver Lavoie (2014) para una exhaustiva exposición sobre el dinero, el crédito y los Bancos Centrales desde una perspectiva postkeynesiana. Nos parece interesante también que el lector tenga presente el ensayo realizado sobre la perspectiva monetaria keynesiana por Kaldor y Trevithik (1981). Véase también el trabajo seminal de Moore (1988).

${ }^{31}$ Como señala Lavoie (2001), la idea de una economía monetizada está asociada al principio de demanda efectiva, pues es difícil imaginar una función de inversión independiente fuera de una economía monetizada. De hecho, los postkeynesianos advierten de que a corto plazo la ruta real tomada por la economía tiene un impacto en los determinantes de la oferta a largo plazo.

${ }^{32}$ La LTGD ignora por principio la influencia que las variaciones del ejército de reserva puedan tener sobre la demanda efectiva de bienes de consumo, como se ha puesto de manifiesto en Halévi y Taouil (2005).
} 
Con todo, el renacimiento del enfoque de dinero endógeno, tal y como lo ha desarrollado la economía post-keynesiana, parte de escritos posteriores a la Teoría General (Keynes 1937a, Keynes 1937b) ${ }^{33}$, obra que desde su publicación ha provocado gran polémica sobre qué constituye su verdadero núcleo analítico dando lugar a interpretaciones walrasianas y no walrasianas de la misma. En estos artículos Keynes tiene en cuenta el papel de la financiación para el crecimiento de la demanda agregada, cuestión que ya tendría presente en los borradores de 1933 de la Teoría General donde exponía las diferencias entre una economía neutral y una economía empresarial, y utilizaba un esquema similar al de Marx (Keynes, 1982) que podría haber ahorrado muchas controversias que surgieron de la versión final de la Teoría General (Keynes, 1936). Así quedaban expuestas las semillas para el desarrollo de una Teoría Monetaria de Producción, en contraposición a lo que se ha denominado "Economía de Intercambios Reales", en referencia a una economía de trueque donde el dinero no es más que una unidad de cuenta. ${ }^{34}$.

El análisis de la endogeneidad de la oferta monetaria puede ser planteado en tres niveles: primero, los vínculos entre empresas y bancos para la financiación de la producción; ${ }^{35}$ segundo, la relación entre el Banco Central y los bancos ${ }^{36}$ y tercero, la relación entre el Estado y el Banco Central. ${ }^{37}$

Debe señalarse que únicamente nos fijamos en este trabajo en el proceso de "flujo" monetario asociado a la financiación inicial del circuito monetario, donde se produce la creación de dinero en función de las expectativas que llevan a los empresarios a incrementar su producción, y no en el proceso de "reflujo" asociado a la operación de saldar las deudas contraídas, relacionada con aspectos como la lucha por la distribución del excedente y la realización de los beneficios. Aunque es cierto que una fase no es independiente de la otra.

Comprender el funcionamiento de una Economía Monetaria de Producción implica reconocer la función básica de los bancos privados. Las empresas emprenden la producción contratando trabajadores gracias al crédito bancario. Esta deuda creada con la concesión de crédito supone la emisión de dinero y por tanto el aumento de la oferta monetaria. La creación por los bancos de los recursos financieros exigidos como condición previa a la puesta en marcha de la producción invierte la causalidad expuesta en la economía neoclásica entre ahorro e inversión. Los préstamos crean los depósitos y por tanto, no hay necesidad de que los bancos tengan depósitos previos basados en reservas o en oro, con el fin de extender préstamos.

Aunque los bancos no se vean restringidos por una determinada oferta de fondos prestables en función del ahorro de los agentes económicos, esto no significa que no exista limitación alguna sobre el crédito que conceden los bancos. La concesión de créditos dependerá, fundamentalmente, de aspectos relacionados con la incertidumbre en el futuro, como la solvencia de los prestatarios, las preferencias del público y el grado de liquidez deseado por los bancos. ${ }^{38}$.

En un segundo nivel, tenemos la relación entre el Banco Central y los bancos. No es necesario que los bancos guarden reservas excedentarias para la creación de dinero nuevo. Otra vez tenemos una relación que se invierte con respecto a lo expuesto por la teoría neoclásica. El dinero bancario es una fracción de los créditos y no un múltiplo de la cantidad de reservas. Una vez los bancos han emitido los créditos y creado dinero en función de las necesidades de la economía, la autoridad monetaria proporcionará las reservas demandadas para mantener el tipo de interés de referencia, que es fijado día a día por el Banco Central para hacer converger las variables económicas al nivel deseado. Afirmar que los bancos centrales tienen un poder discrecional para fijar el tipo de interés acarrea el sostener que, cuando la demanda de crédito aumen-

\footnotetext{
${ }^{33}$ Smithin (2013) analiza el pensamiento de Keynes en los aspectos clave de una Teoría Monetaria.

${ }^{34}$ Schumpeter (1912) puede ser considerado el padre de tal enfoque, dada su concepción del papel de la creación de crédito en la financiación de una economía de emprendedores.

${ }^{35}$ Expuesto fundamentalmente por autores de la Teoría del Circuito Monetario. Ver Graziani (2003).

${ }^{36}$ La relación entre los bancos y el Banco Central es discutida ampliamente en los debates entre los denominados "horizontalistas" y "estructuralistas". Para una evaluación de los mismos, ver Palley (2013).

${ }^{37}$ Wray (2015) presenta los fundamentos básicos de lo que se conoce como Teoría Monetaria Moderna.

${ }^{38}$ Chick y Dow (2013) exponen los puntos clave de los diferentes enfoques sobre el dinero en la economía postkeynesiana y sus puntos de confrontación, centrándose especialmente en la preferencia por la liquidez de los bancos.
} 
ta, no están obligados a elevar el tipo de interés de referencia, sino que éste se corresponderá con la respuesta que desee dar la autoridad monetaria. ${ }^{39}$

Otra forma de crear dinero es mediante el gasto público del Estado. Los bonos emitidos por el Tesoro y admitidos por los bancos juegan un papel similar al de los préstamos realizados por los bancos a las empresas, puesto que los ingresos, fundamentalmente procedentes de los impuestos, deben ser considerados como una renta contingente a la percepción de renta por parte de los agentes privados. Por tanto, son determinados una vez se ha realizado el gasto por parte de las empresas, las economías domésticas y el propio Estado.

El Estado necesita gastar antes de recaudar, y estos gastos afectan al nivel global de renta influyendo en las decisiones de gasto del sector privado, lo que a su vez afecta a las reservas de los bancos. A fin de asegurarse el mantenimiento del tipo de interés de referencia, para que éste no baje, el Banco Central debe drenar las reservas excedentarias acumuladas por el sector bancario, suponiendo que el Gobierno incurre en déficit presupuestario, mediante la venta de bonos del Tesoro. ${ }^{40}$

Resumiendo, desde el punto de vista post-keynesiano que contempla la oferta monetaria como endógena se rechazan las siguientes hipótesis surgidas de la teoría neoclásica: (i) la cantidad de reservas en manos de los bancos determina la oferta de dinero, que opera a través del multiplicador monetario; (ii) existe un tipo de interés natural dependiente del mercado; y (iii) los déficits presupuestarios del Gobierno, financiados a través de la emisión de bonos del Tesoro, ejercen una presión al alza de los tipos de interés debido al efecto expulsión tal como sugiere la teoría de los fondos prestables.

La lectura final es, por tanto, evidente. La capacidad del sistema bancario de crear dinero para financiar los incrementos de flujos de producción es un elemento esencial en el funcionamiento de una economía monetaria de producción, como es el capitalismo en que vivimos.

\section{Reflexiones}

Desde los economistas clásicos, el análisis real se alza como eje dominante en la manera de hacer economía. La teoría de precios relativos definidos como tasas de sustitución entre bienes físicamente determinados, antes e independientemente de cualquier referencia al dinero, es la base sobre la cual se erige la teoría del equilibrio general, núcleo irrebatible dentro de la economía neoclásica. Solo posteriormente se introdujo el dinero como un "velo", un dispositivo neutral que no afecta en nada a la estructura y niveles de precios relativos y a las funciones de oferta y demanda que les están asociadas.

Hemos visto que hubo oportunidades en la historia de cambiar el enfoque analítico y de orientar el mismo hacia la concepción de una economía monetaria de producción. Sin embargo, la defensa de la teoría cuantitativa por la ortodoxia durante el siglo XIX y la posterior anulación de la revolución de Keynes en el XX lo impidieron.

La defensa de la Teoría Cuantitativa por parte de los economistas clásicos se fundamentó principalmente en las restricciones reales inherentes al mundo en el que vivieron, las cuales quedan reflejadas en el pesimismo de Ricardo (1817), cuya vigorosa campaña para la revocación de la Ley de Granos en Inglaterra se basó, fundamentalmente, en intentar salvar de alguna manera estos obstáculos impuestos por los rendimientos decrecientes de la tierra para retrasar el estado estacionario.

Por su parte, la neutralización de la revolución comenzada por Keynes se consumó cuando autores como Hicks consiguieron desproveer a la obra del economista de Cambridge de sus elementos más críticos. El propio Hicks reconoció un sesgo en su visión de la Teoría General, admitiendo que había omitido erróneamente la discusión sobre la incertidumbre o las expectativas, dejando sin sentido a la preferencia por la liquidez tal como queda representada en el mode-

\footnotetext{
${ }^{39}$ Ver Moore (2006) para una explicación sobre la fijación del tipo de interés de referencia por los Bancos Centrales.

${ }^{40}$ Ver Bougrine y Seccareccia (2006) para una exposición de los flujos de fondos entre el Banco Central, Tesoro y sector privado.
} 
lo IS-LM (Hicks, 1980). Igualmente grave es la ausencia del dinero y del crédito en este modelo, tal y como ocurre en el conjunto de la teoría neoclásica, que podría haberse orientado por otros caminos más prometedores en cuanto a su utilidad prescriptiva si la obra de Fisher y el proceso acumulativo descrito por Wicksell hubiesen sido tomados en consideración.

Hubo que esperar hasta Minsky (1986) para incluir las deudas en un esquema coherente que considerase el papel de los bancos en una economía capitalista de crédito puro, con efectos de retroalimentación que alejan la economía de su equilibrio, tal y como se expone en su "hipótesis de inestabilidad financiera".

En nuestro criterio, el análisis de la creación de dinero endógeno está fuertemente ligado al funcionamiento y la evolución del sistema económico y de las instituciones. Las innovaciones en el sistema bancario se originaron de las necesidades que la expansión de la producción y la inversión en una economía en crecimiento, desde unos primeros estadios donde una oferta monetaria inelástica provocaba una tendencia de la economía a pararse antes de llegar al pleno empleo. La idea de que el ahorro es una condición previa a la inversión, tal y como sostiene la visión del negocio bancario como mero conductor del ahorro, se vuelve equivocada cuando el sistema bancario alcanza la etapa en la cual sus pasivos se convierten en los medios de pago preponderantes, ${ }^{41}$ de manera que los bancos en su conjunto pueden expandir el crédito hasta un múltiplo de sus reservas.

Un análisis complejo que pretenda renovar el estudio de los fenómenos del crecimiento y de las fluctuaciones debe abandonar la dicotomía clásica e integrar las cuestiones en torno al dinero y las relaciones de endeudamiento. Ello supone analizar el mundo real con sus características propias, en lugar de un mundo idealizado al que intentar ajustarse, tal como plantean los relativamente recientes esfuerzos de la teoría del circuito schmittiana de ajustar el análisis walrasiano al proceso de producción (Schmitt, 1984). Este planteamiento oscurece el tratamiento de la endogeneidad del dinero y su no neutralidad partiendo de una definición del dinero como forma numérica del producto; esto es, un numerario. Con el rechazo de que el dinero sea un activo financiero, los bancos no actuarían como creadores de poder de compra con la emisión del mismo, sino que delimitarían su actividad a una intermediación financiera intertemporal en la que los depósitos bancarios medirían el poder de compra de los titulares de las rentas de nueva formación de la economía, es decir, de la nueva producción hecha por los trabajadores, llevándonos de nuevo a la máxima de Say de que los productos compran productos.

Nuevamente, vemos aquí la monetización de la producción que nos lleva al callejón de una economía de trueque que queda lejos de la realidad de la actividad bancaria, y no al desarrollo de una Economía Monetaria de Producción. El enfoque de la endogeneidad de la oferta monetaria tiene una explicación clara: los gastos previstos son financiados gracias a los créditos concedidos por el sistema bancario, realizándose la financiación de la inversión adicional con la creación de poder de compra. ${ }^{42}$

Creemos que el hecho de que el sector privado no pueda iniciar cambios en la oferta de dinero, o lo haga cuando quizás ello no sea lo pertinente, presenta unos desafíos que debemos abordar repensando el papel del dinero en los modelos tradicionales. Avanzar hacia una síntesis de los trabajos de Marx, Schumpeter, Keynes y Minsky, tal y como intentan de alguna manera promover los diversos desarrollos de teoría monetaria inmersos en la escuela post-keynesiana, es terreno fructífero para una teoría económica más útil en la prescripción de soluciones que, como vemos en situaciones turbulentas como la actual, la economía neoclásica no parece poder proveer.

\section{Bibliografía}

Alonso, Miguel A.; Bagus, Philipp; y Rallo, Juan R. 2011. Teorías del ciclo económico: principales contribuciones y análisis a la luz de las aportaciones de la Escuela Austríaca de Econo-

\footnotetext{
${ }^{41}$ Véase el magnífico trabajo de Victoria Chick sobre la evolución por etapas del sistema bancario (Chick, 1986).

${ }^{42}$ Este aspecto de la emisión de dinero bancaria se trata con profusión en Kalecki (1990).
} 
mía. Información Comercial Española, 858 (Monográfico "Tendencias y nuevos desarrollos de la Teoría económica), 71-87.

Anisi, David. 2005. La macroeconomía al comienzo del siglo XXI: una reflexión sobre el uso y posterior abandono del llamado keynesianismo. Principios: Estudios de Economía Política, 1, 37-55.

Arestis, Philip; y Sawyer, Malcom. 2008. A critical reconsideration of the foundations of monetary policy in the new consensus macroeconomics framework. Cambridge Journal of Economics, 32(5), 761-779.

Arnon, Arie. 2011. Monetary theory and policy from Hume and Smith to Wicksell: Money, cred$i t$, and the economy. Cambridge, Cambridge University Press.

Blanchard, Olivier. 2009. The State of Macro. Annual Review of Economics, 1(1), 209-228.

Blaug, Mark. 1962. Economic Theory in Retrospect, Homewood, Illinois: Richard D. Irwin. Versión en español de Mark Blaug, 1985. Teoría económica en retrospección. México, Fondo de la Cultura Económica.

Boermans, Martijn A.; y Moore, Basil J. 2009. Locked-in and Sticky Textbooks: Mainstream Teaching of the Money Supply Process, 14845. Munich, University Library of Munich.

Bougrine, Hassan y Seccareccia, Mario. 2006. El papel de los impuestos en la economía nacional. En Pierre Piégay y Louis-Philippe Rochon (eds.), Teorías monetarias poskeynesianas. Madrid, Akal.

Cantillon, Richard. 2011 [1755]. Essai sur la Nature du Commerce en Général, París, Institut Coppet, edición de Stéphane Couvreur.

Chick, Victoria. 1990. La macroeconomía según Keynes. Madrid, Alianza Editorial.

-1986. The evolution of the banking system and the theory of saving, investment and interest, Economies et Sociétés, 20, (Monnaie et Production, 3), 11-26.

Chick, Victoria; y Dow, Sheila. 2013. Post-Keynesian Theories of Money and Credit: Conflicts and (some) Resolutions. In Geoffrey Harcourt and Peter Kriesler (eds.), The Oxford Handbook of Post-Keynesian Economics, Oxford, volumen 1: Theory and Origins.

Deleplace, Ghislain. 2008. La absorción de la Macroeconomía por la Microeconomía. Lecturas de Economía, (69), 245-298.

De Roover, Raymond. 1955. Scholastic economics: survival and lasting influence from the sixteenth century to Adam Smith. The Quarterly Journal of Economics, 69 (2), 161-190.

De Roover, Raymond; y Kirshner, Julius. 1974. Business, banking, and economic thought in late medieval and early modern Europe. Chicago, University of Chicago Press.

Fanfani, Amintore. 1933. Le Origini dello spirito capitalistico in Italia. Milán, Vita e pensiero.

Fontana, Giuseppe; y Passarella, Marco V. 2014. Aggregate Demand, Money and Finance within the New Consensus Macroeconomics: a Critical Appraisal. FESSUD Project: Working Paper Series.

Fisher, Irving. 1911.The purchasing power of money. New York, Macmillan

Friedman, Milton. 1969. The Optimum Quantity of Money. London, Macmillan.

- 1987. Quantity theory of money. En John Eatwell, Murray Milgate, and Peter Newman, The New Palgrave: A Dictionary of Economics. New York, Macmillan, 3-20.

Friedman, Milton; y Kuttner, Kenneth N. 2010. Implementation of Monetary Policy: How Do Central Banks Set Interest Rates? NBER Working Paper, 16165.

Galí, Jordi; y Gertler, Mark. 2007. Macroeconomic Modeling for Monetary Policy Evaluation. The Journal of Economic Perspectives, 21(4), 25-45.

Giacomin, Alberto. 2007. Paper money: a reassessment of Adam Smith's views. En Alberto Giacomin y Maria Cristina Marcuzzo (eds.), Money and Markets: A doctrinal approach. Londres, Routledge.

Giraldo, Andrés F. 2006. La neutralidad del dinero y la dicotomía clásica en la macroeconomía. Cuadernos de economía, 25(45), 75-93.

Graziani, Augusto. 2003. The monetary theory of production. Cambridge, Cambridge University Press. 
Halévi, Joseph; y Taouil, Réduane. 2005. La exogeneidad de la inversión: de las leyes sistémicas de acumulación y crecimiento a las condiciones de demanda efectiva. En Mark Setterfield (ed.), La economía del crecimiento dirigido por la demanda. Madrid, Akal.

Häring, Norbert. 2013. The veil of deception over money: how central bankers and textbooks distort the nature of banking and central banking. Real-world Economics Review, 63, 2-18.

Hayek, Friedrich A. 1931. Prices and Production. Londres, Routledge.

Hein, Eckhard. 2008. Money, Distribution and Capital Accumulation. New York, Palgrave MacMillan.

Hicks, John. 1937. Mr. Keynes and the "Classics": a suggested interpretation. Econometrica, 5(2), 147-159.

- 1980. IS-LM: an explanation. Journal of Post Keynesian Economics, 3(2), 139-154.

Hume, David. 1752. Political discourses. Edinburgh, A. Kincaid and A. Donaldson.

Jijón, Armando. 2000. Breve reseña histórica sobre la literatura relacionada con la neutralidad monetaria. Cuestiones Económicas, 16 (2), 193-2015.

Kaldor, Nicholas. 1970. The new monetarism. Lloyds Bank Review, 97(1), 1-18.

Kaldor, Nicholas; y Trevithick, James. 1981. A Keynesian Perspective on Money. Lloyds Bank Review, 139, enero, 1-19.

Kalecki, Michal. 1990. Capitalism: Business Cycles and Full Employment. In Jerzy Osiatynski (ed.), Collected Works of Michal Kalecki, Oxford, Clarendor Press, vol.1.

Keen, Steve. 2015. La economía desenmascarada. Madrid, Capitán Swing.

Keynes, John M. 1936. The General Theory of Interest, Employment and Money. London, Macmillan Cambridge University Press.

- 1937a. Alternative theories of the rate of interest. In Harry G. Johnson and Donald E. Moggridge (eds.), The Collected Writings of John Maynard Keynes, London, Macmillan Cambridge University Press, vol. XIV.

- 1937b. The "ex ante" theory of the rate of interest. In Harry G. Johnson and Donald E. Moggridge (eds.), The Collected Writings of John Maynard Keynes, London, Macmillan Cambridge University Press, vol. XIV.

- 1982. The Collected Writings of John Maynard Keynes. In Harry G. Johnson and Donald E. Moggridge (eds.), London, Macmillan Cambridge University Press, vol. XXVIII.

King, John E. 2009. Historia de la economía poskeynesiana desde 1936. Madrid, Akal.

- 2015. Advanced Introduction to Post Keynesian Economics. Cheltenham, Edward Elgar.

Lavoie, Marc. 1984. The Endogenous Flow of Credit and the Post Keynesian Theory of Money. Journal of Economic Issue, 18 (3), 771-797.

- 2006. Introduction to post-Keynesian economics. Nueva York, Palgrave Macmillan.

- 2011. The history and methods of post-Keynesian economics. In Eckhard Hein and Engelbert. Stockhammer (eds.), A modern guide to Keynesian macroeconomics and economic policies. Cheltenham, Edward Elgar.

- 2014. Post-Keynesian Economics: New Foundations. Cheltenham, Edward Elgar.

Law, John. 1705. Money and Trade Considered with a Proposal for Supplying the Nation with Money. Edinburgo, R.\&A. Foulis.

Lindner, Fabian. 2015. Does Saving Increase the Supply of Credit? A Critique of Loanable Funds Theory, World Economic Review, 4.

Locke, John. 1691. Someconsiderations of the consequences of the lowering of interest, and raising the value of money. Londres, Awnsham and John Churchill.

Marx, Karl. 1978 [1867]. El Capital. Crítica de la Economía Política. Madrid, Siglo XXI, Libro I.

- 1979 [1885]. El Capital. Crítica de la Economía Política. Madrid, Siglo XXI, Libro II.

McLeay, M.; Radia, A. y Thomas, R. 2014.Money creation in the modern economy. Bank of England Quarterly Bulletin, Q1.

Minsky, Hyman. 1986.Stabilizing and Unstable Economy. Nueva York, Yale University Press.

Moore, Basil J. 1988.Horizontalists and Verticalists: The Macroeconomics of Credit Money. Cambridge, Cambridge University Press. 
— 2006. La endogeneidad de la oferta de dinero: ¿se fija el precio o la cantidad de reservas? En Pierre Piégay y Loius-Philippe Rochon (eds.), Teorías monetarias poskeynesianas. Madrid, Akal.

Ospina, Armando G.; y García, Alejandro T. 2009. Una revisión de los principales desarrollos de la teoría económica neoclásica en las últimas décadas y sus perspectivas. Revista Páginas, 83, 17-38.

Palley, Thomas I. 2013. Horizontalists, verticalists, and structuralists: the theory of endogenous money reassessed. Review of Keynesian Economics, 4, 406-424.

Quesnay, François. 1985 [1765] Derecho natural, Madrid, Centro de Estudios Constitucionales, versión en castellano contenida en Escritos Fisiocráticos.

Ricardo, David. 1817. On the Principles of Political Economy and Taxation. Londres, John Murray.

Rogers, Colin. 1989. Money, Interest and Capital: a study in the foundations of monetary theory. Cambridge, Cambridge University Press.

- 2013. The Scientific Illusion of New Keynesian Monetary Theory. In Geoffrey Harcourt and Peter Kriesler (eds.), The Oxford Handbook of Postkeynesian Economics, Oxford Handbooks, vol. 1.

Roncaglia, Alessandro. 2006. La riqueza de las ideas. Una historia del pensamiento económico. Zaragoza, Prensas Universitarias de Zaragoza (Traducción de Jordi Pascual Escutia).

Say, Jean-Baptiste. 1803. Traité d'économie politique, Traité d'économie politique ou simple exposition de la manièredont se forment, se distribuent et se composent les richesses. París, Crapelet.

Schmitt, Bernard. 1984. Inflation, chômageet malformations du capital: macroéconomiequantique. Suisse, Albeuve, Castella; Paris, Économica.

Schumpeter, Joseph A. 1912. Theorie der wirtschaftlichen Entwickung. Leipzig, Duncker \& Humblot. Traducción al ingles publicada en 1934, The Theory of Economic Development. Cambridge, MA, Harvard University Press.

- 1954. History of Economic Analysis. Londres, Allen Unwin. Versión española (2012), Historia del análisis económico. Barcelona, Ariel.

Segura, Julio; y Rodríguez Braun, Carlos. 1998. La economía en sus textos. Madrid, Taurus.

Smith, Adam. 1776.An Inquiry into the Nature and Causes of the Wealth of Nations, London: W. Strahan and T. Cadell.

Smithin, John. 2013. Keynes's theories of money and banking in the Treatise and The General Theory. Review of Keynesian Economics, 1(2), 242-256.

Taylor, Lester D. 2008. Capital, Accumulation and Money: An Integration of Capital, Growth and Monetary Theory. Springer ( $2^{\mathrm{a}}$ ed.).

Thornton, Henry. 1802. An enquiry into the nature and effects of the paper credit of Great Britain. Londres, J. Hatchard, vol. 595(6).

Turgot, Anne Robert Jacques. 1766. Réflexions sur la formation et la distribution des richesses. Versión en castellano por Eduardo Escartín González (2003). Estudio y traducción de la obra: Reflexiones sobre la formación y la distribución de las riquezas de R. J. Turgot. Sevilla, Secretariado de Publicaciones de la Universidad de Sevilla.

Woodford, Michael. 2009. Convergence in macroeconomics: elements of the new synthesis. American economic journal: macroeconomics, 1(1), 267-279.

Walras, Leon. 2003 [1877]. Elements of pure economics or the theory of social wealth. London, Routledge.

Wicksell, Knut. 1898. Geldzins und Güterpreise. Jena, G. Fischer. Traducción de la obra al inglés en Richard F. Kahn (1936). Interest and Prices. Londres, Macmillan.

Wray, Randall. 2015. Teoría Monetaria Moderna. Berlín, Lola books. 